\title{
Dimensional changes of commercial and novel polyvinyl siloxane impression materials following sodium hypochlorite disinfection
}

\author{
Shahab Ud Din ${ }^{\text {Corresp., } 1}$, Muhammad Sajid ${ }^{2}$, Asfia Saeed ${ }^{2}$, Farooq Ahmad Chaudhary ${ }^{1}$, Mohammad Khursheed Alam \\ , Juneda Sarfraz ${ }^{1}$, Bilal Ahmed ${ }^{1}$, Mangala Patel $^{4}$ \\ ${ }^{1}$ School of Dentistry (SOD), Federal Medical Teaching Institution (FMTI)/PIMS, Shaheed Zulfiqar Ali Bhutto Medical University (SZABMU), Islamabad, \\ Pakistan \\ 2 Department of Dental Materials, Islamabad Medical \& Dental College, Islamabad, Pakistan \\ 3 Preventive Dentistry Department, College of Dentistry, Jouf University, 72345, Sakaka, Saudi Arabia \\ 4 Centre for Oral Bioengineering (Dental Physical Sciences Unit), Bart's and The London School of Medicine andDentistry, Queen Mary University of \\ London, London, United Kingdom \\ Corresponding Author: Shahab Ud Din \\ Email address: drshahab728@hotmail.com
}

Background: Dental impressions are used to record anatomy of teeth and surrounding oral structures. Impression materials becomes contaminated with saliva and blood leading to health hazards. Disinfection reduces cross infection but may have negative impact on dimensional stability of materials. Objective: Comparatively evaluate linear dimensional changes of synthesized Tetra-functional (dimethylsilyl) orthosilicate (TFDMOS) containing Polyvinylsiloxane (PVS) impressions following sodium hypochlorite disinfection. Methods: Percentage dimensional changes of three commercial PVS (Elite HD Monophase, Extrude and Aquasil Ultra Monophase) and five experimental PVS impression materials were measured. Experimental material contained novel cross-linking agent (TFDMOS) and a non-ionic surfactant (Rhodasurf CET-2) that is Exp-A (without TFDMOS), Exp-B (with TFDMOS), Exp-C (TFDMOS+ 2\% Rhodasurf CET-2), Exp-D (TFDMOS+ 2.5\% Rhodasurf CET-2) Exp-E (TFDMOS+ 3\% Rhodasurf CET-2). Samples were made using rectangular stainless-steel molds $\left(40 \times 10 \times 3 \mathrm{~mm}^{3}\right)$ and linear dimensional changes were measured using a calibrated travelling microscope at 10x magnification after immersion in distilled water (D.W) and $1 \%$ Sodium Hypochlorite solution at two different time intervals i.e., 30 minutes and 24 hours. Results: Samples immersed in $1 \% \mathrm{NaOCl}$ showed significant $\left(\mathrm{p}^{<0.05)}\right.$ dimensional changes after 30 minutes of immersion. Exp-E showed significantly greater dimensional changes than their control (Exp-A and Exp-B). In distilled water, there were no significant difference among the tested materials. Aquasil exhibited highest expansion $(0.06 \%)$ in both solutions. At 24 hours, among the commercial materials, Extrude had the greatest expansion followed by Aquasil and Elite in DW while Aquasil showed the greatest 
expansion followed by Extrude and Elite in $\mathrm{NaOCl}$. Conclusion: Experimental PVS had linear dimensional changes within the ISO 4823; 2015 recommended range. However, extended immersion can negatively affect the linear dimensions. 


\section{Dimensional changes of commercial and novel polyvinyl}

2 siloxane impression materials following sodium hypochlorite

\section{3 disinfection}

4

5 Shahab Ud Din ${ }^{1}$, Muhammad Sajid ${ }^{2}$, Asfia Saeed ${ }^{2}$, Farooq Ahmad Chaudhary ${ }^{1}$, Mohammad

6 Khursheed Alam ${ }^{3}$, Juneda Sarfraz ${ }^{1}$, Bilal Ahmed ${ }^{1}$, Mangala Patel ${ }^{4}$

7

$8{ }^{1}$ School of Dentistry (SOD), Federal Medical Teaching Institution (FMTI)/PIMS, Shaheed

9 Zulfiqar Ali Bhutto Medical University (SZABMU), Islamabad, Pakistan.

$10{ }^{2}$ Department of Dental Materials, Islamabad Medical \& Dental College, Islamabad, Pakistan.

$11{ }^{3}$ Preventive Dentistry Department, College of Dentistry, Jouf University, 72345 Sakaka,

12 Saudi Arabia.

$13{ }^{4}$ Centre for Oral Bioengineering (Dental Physical Sciences Unit), Bart's and The London

14 School of Medicine and Dentistry, Queen Mary University of London, UK.

Corresponding Author:

Shahab Ud Din ${ }^{1}$

School of Dentistry (SOD), Federal Medical Teaching Institution (FMTI)/PIMS, Shaheed

Zulfiqar Ali Bhutto Medical University (SZABMU), Islamabad, Pakistan.

Email address: drshahab728@hotmail.com 


\section{Abstract}

27 Background: Dental impressions are used to record anatomy of teeth and surrounding oral structures. Impression materials becomes contaminated with saliva and blood leading to health hazards. Disinfection reduces cross infection but may have negative impact on dimensional stability of materials.

Objective: Comparatively evaluate linear dimensional changes of synthesized Tetra-functional (dimethylsilyl) orthosilicate (TFDMOS) containing Polyvinylsiloxane (PVS) impressions following sodium hypochlorite disinfection.

Methods: Percentage dimensional changes of three commercial PVS (Elite HD Monophase, Extrude and Aquasil Ultra Monophase) and five experimental PVS impression materials were measured. Experimental material contained novel cross-linking agent (TFDMOS) and a non-ionic surfactant (Rhodasurf CET-2) that is Exp-A (without TFDMOS), Exp-B (with TFDMOS), Exp-C (TFDMOS $+2 \%$ Rhodasurf CET-2), Exp-D (TFDMOS $+2.5 \%$ Rhodasurf CET-2) Exp-E (TFDMOS $+3 \%$ Rhodasurf CET-2). Samples were made using rectangular stainless-steel molds $\left(40 \times 10 \times 3 \mathrm{~mm}^{3}\right)$ and linear dimensional changes were measured using a calibrated travelling microscope at 10x magnification after immersion in distilled water (D.W) and $1 \%$ Sodium Hypochlorite solution at two different time intervals i.e., 30 minutes and 24 hours.

Results: Samples immersed in $1 \% \mathrm{NaOCl}$ showed significant $(\mathrm{p}<0.05)$ dimensional changes after 30 minutes of immersion. Exp-E showed significantly greater dimensional changes than their control (Exp-A and Exp-B). In distilled water, there were no significant difference among the tested materials. Aquasil exhibited highest expansion (0.06\%) in both solutions. At 24 hours, 
47 among the commercial materials, Extrude had the greatest expansion followed by Aquasil and

48 Elite in DW while Aquasil showed the greatest expansion followed by Extrude and Elite in $\mathrm{NaOCl}$.

49 Conclusion: Experimental PVS had linear dimensional changes within the ISO 4823; 2015

50 recommended range. However, extended immersion can negatively affect the linear dimensions.

\section{Introduction}

53 Dental impressions are used to accurately record and reproduce the shape, relationship of teeth and 54 surrounding oral structures (Kumari \& Nandeeshwar 2015). However, during impression making, 55 the material becomes contaminated with saliva and often with blood which may pose health hazard 56 to the dental team (Ud Din et al. 2018). Different methods of disinfection including spraying and 57 immersion of impression material are commonly employed (Khan 2018). However, American 58 Dental Association (ADA) recommends the immersion technique because it allows direct contact 59 of disinfectant solutions with all surfaces of the impression (Samra \& Bhide 2018). Disinfection 60 through immersion reduces the risk of cross infection, yet alteration in dimensions of impression 61 materials and negative impact on quality of the resulting cast has often been reported (Guiraldo et 62 al. 2012; Martin et al. 2007). This might result in dimensional changes in dental prosthesis and 63 ultimately affect the fit of the final restoration (Asopa et al. 2020). Iwasaki et al. (2016) reported 64 marked changes in the dimensions of alginate impressions due to water sorption after prolonged 65 immersion in a disinfectant solution (Iwasaki et al. 2016). Similarly, Babiker et al. (2018) reported 66 significant dimensional changes of gypsum casts obtained from irreversible hydrocolloids after 67 disinfection by $\mathrm{NaOCl}$ immersion (Babiker et al. 2018).

68 The recommended products for disinfection of the impression materials are sodium hypochlorite, 69 chlorhexidine, glutaraldehyde and iodine agents (Correia-Sousa et. al, 2013). Among numerous 
70 disinfectants available, sodium hypochlorite has been recommended by the ADA and

71 Environmental Protection Agency (EPA) for the disinfection of impressions due to its low cost

72 and broad spectrum antimicrobial activity (Correia-Sousa et al. 2013; Khan and Mushtaq 2018).

73 A study by Al-Enazi and Naik on the efficacy of $1 \%$ sodium hypochlorite and $2 \%$ glutaraldehyde

74 disinfectant sprays on impression materials (alginate and addition silicone rubber-based

75 impression material) established that $1 \%$ sodium hypochlorite yielded better results (Al Enazi and

76 Naik 2016).

77

78 Recently polyvinylsiloxane has gained popularity due to its ability to precisely record tissue

79 details, excellent physical properties and easy manipulation, and is preferred for making final

80 impressions of fixed dental prosthesis (Azevedo et al. 2019). Nevertheless, these materials have

81 lower tear strength and percentage elongation compared to polysulphide. Additionally, the

82 hydrophobic nature of the material demands a dry field of operation to accurately record fine tissue

83 details (Ud Din et al. 2018).

84 Different researchers have incorporated surfactant to the material composition to synthesize 85 hydrophilic addition silicones, but limited literature is available on methods to improve the tear 86 strength of the material (Lee et al. 2004). Formerly, we have carried out synthesis of novel 87 TFDMOS containing polyvinyl siloxane with a non-ionic surfactant (Rhodasurf CET-2) to 88 improve the tear strength and hydrophilicity of conventional addition silicones. It was observed 89 that experimental formulation had significantly higher tear strength compared to commercial 90 material and that tear strength was directly proportional to the concentration of non-ionic surfactant

91 (Din et al. 2018). Considering the variation in material properties corresponding to the composition 92 of the material, the effect of non-ionic surfactant on dimensional stability of materials especially 93 after disinfection needed further exploration. It was hypothesized that the experimental 
94 formulations have higher water sorption due to the presence of non-ionic surfactant, resulting in

95 noticeable dimensional changes.

96 Extensive use of polyvinylsiloxane in dentistry and varieties of formulation available necessitated

97 a comprehensive comparison of material particularly in terms of dimensional changes following

98 long term disinfection. Therefore, the objective of this study was two-fold including formulation

99 of experimental polyvinylsiloxane containing TFDMOS and evaluation of the influence of storage

100 media that is distilled water and 1\% sodium hypochlorite on the linear dimensional changes over

101 the period of 24 hours, in commercial and synthesized PVS impression materials.

102

103 Materials \& Methods

104 In this in-vitro experimental study, three commercially available medium bodied PVS materials, 105 Aquasil (Ultra, Dentsply, USA), Elite HD Monophase (Zhermack, Italy) and Extrude (Kerr, USA)

106 were selected. In addition, five experimental PVS materials were prepared as base and catalyst 107 paste by weighing all components using a four beam balance (Mettler, Toledo Ltd, Model AG204, $108 \mathrm{UK}$ ) as already mentioned in the published part of the study (Ud Din et al. 2018). The composition 109 of experimental materials is illustrated in Table 1. To prevent premature polymerisation, equal 110 amount of the prepared material was weighed with a four beam balance and was packed into 111 separate compartments of an auto-mixing cartridge and stored at $4^{\circ} \mathrm{C}$. Exp-A served as a control

112 for Exp-B, while Exp-B was used as a control for Exp-C, D and E. Rhodasurf CET-2 (non-ionic 113 surfactant) was incorporated in the base paste of Exp-B at 2, 2.5 and 3\% to formulate Exp C, D 114 and E respectively.

115 A total of eighty samples ( $\mathrm{n}=10$ for each material) were prepared by introducing the material from 116 automixing syringe into the preformed stainless steel mold ( $\left.40 \times 10 \times 2 \mathrm{~mm}^{3}\right)$ sandwiched between 
117 two metal plates lined by acetate paper. The assembly was placed under a hydraulic press

118 (MESTRA MOD-030350, Talleres Mestraitua, Spain). Commercial material was allowed to

119 polymerize for the time specified by the manufacturers, while experimental formulation were

120 polymerized for 10 minutes (Ud Din et al. 2018; Din et al. 2018).

121

122 After polymerization, the material was removed from the mold and the specimens were divided 123 into 2 test groups, each consisting of 5 samples from each material. Group 1 was immersed in 1\% $124 \mathrm{NaOCl}$ and Group 2 in distilled water solution for 24 hours at $23 \pm 1^{\circ} \mathrm{C}$ in an oven (Qualicool 125 incubators, LTE Scientific Ltd). At predetermined time periods, which were 10 and 30 min 126 (replicate at-office disinfection), and $60 \mathrm{~min}$ and 24 hours ( simulating the transportation time for 127 the impression to reach the dental laboratory) each sample was removed from the respective liquid 128 and blot dried. Linear dimensional changes were recorded using a calibrated travelling microscope 129 (Chesterman, Sheffield, England) by measuring the distance between the fixed edge and free end 130 of the sample (movable pin) at 10x magnification. Data were presented as mean and standard 131 deviation using SPSS Version 22 (Armonk NY IBM Corp). Analysis of variance with post hoc 132 Tukey's test was performed to statistically compare all PVS materials at different time points and $133 \mathrm{p}$ value of 0.05 was considered as significant.

\section{Results}

136 Figure 1 and 2 show percentage linear dimensional changes of impression materials in DW and $1371 \% \mathrm{NaOCl}$ after 24 hours immersion. Aquasil exhibited the highest expansion (0.06\%) in both 138 solutions while Exp-A exhibited the least expansion (0.01\%) in DW. For Group 1, a significant 139 difference $(\mathrm{p} \leq 0.05)$ in dimensions was observed after 30 minutes of immersion. Inter-group 
140 analysis revealed statistically similar dimensional changes among all tested materials, except for

141 Exp-E (Table II). The surfactant modified Exp-E showed significantly $(\mathrm{p} \leq 0.05)$ greater

142 dimensional changes than their control groups (Exp A \& Exp B). Among commercial materials,

143 although no significant changes in dimensions were observed, Aquasil showed the highest

144 expansion followed by Extrude and Elite in $1 \% \mathrm{NaOCl}$ solution.

145 For Group 2 immersed in distilled water, there were no significant differences among the

146 commercial and experimental materials. Extrude had the highest expansion followed by Aquasil

147 and Elite at 24 hours. Exp B showed slightly higher, but statistically similar expansion to Exp-A,

148 while Exp-C, D and E exhibited higher expansion in each immersion solution compared to Exp-B

149 (control) at each interval. Also, expansion of experimental materials was directly related to the

150 concentration of the surfactant.

151

152 Discussion

153 Impression making is an important aspect of prostheses fabrication. In the oral cavity, these 154 materials come in contact with saliva and blood (Khan and Mushtaq 2018). This demands 155 disinfection of materials to prevent cross-infection in dental clinics and hospital. However, it is 156 important that the impressions remain dimensionally stable after disinfection.

157 The results of the present study revealed that immersion of addition silicones impression materials 158 in distilled water (Group 2) had no significant impact on the dimensional stability of the material 159 disinfection of impression with $1 \% \mathrm{NaOCl}$ (Group 1) for 24 hours resulted in expansion of the 160 tested materials. However, the dimensional changes observed were not clinically relevant as the 161 values are within the permitted range of $\leq 0.5 \%$ as recommended by ISO $4823 ; 2015$ (ISO) and 
162 ADA specification 19 (Association 2007); thus, indicating high precision of the experimental 163 material.

164 The limited amount of linear expansion could be attributed to the isotropic expansion of the 165 samples as they adhere to the PTFE trough of the travelling microscope. These findings were 166 supported by Carvalhal et al (2011) who investigated the dimensional changes of polysulphide, 167 polyether, addition and condensation silicone after immersion disinfection with $0.5 \% \mathrm{NaOCl}$ and $1682 \%$ glutaraldehyde. They observed insignificant changes in the linear dimensional of materials and 169 recommended that all synthetic elastomers can be safely disinfected by immersion in $0.5 \% \mathrm{NaOCl}$ 170 and 2\% glutaraldehyde (Carvalhal et al. 2011). In a similar study, Samra and Bhide (2018) 171 observed clinically acceptable dimensional changes for alginate and PVS after disinfection with 172 ultraviolet rays and immersion disinfection with sodium hypochlorite, changes in cross-arch space 173 and inter-abutment distance on gypsum casts were noted for materials disinfected by 174 glutaraldehyde immersion (Samra \& Bhide 2018).

175 Furthermore, Exp-E had significantly greater dimensional changes after immersion disinfection 176 with $\mathrm{NaOCl}$. This was in line with a number of studies that indicated hydrophilic silicones have 177 greater tendency to absorb water and expand (Silva \& Salvador 2004). Results also indicated that 178 longer immersion periods can affect the materials. These findings were similar to those noted by 179 Johnson (1996), who reported long-term dimensional stability of PVS to be unsatisfactory 180 (Thouati et al. 1996). Similarly, Kumari and Nandeeshwar (2015) observed significant difference 181 between non-disinfected and disinfected specimens of PVS and polyether after immersion of 16 182 hours in $0.525 \%$ sodium hypochlorite (Kumari \& Nandeeshwar 2015). On contrary, Nassar et al. 183 found no statistically significant difference between dimensions of vinyl polyether silicone at the 184 time of fabrication and after storage for 1 and 2 weeks in $2.5 \%$ buffered solution of glutaraldehyde 
185 186

and reported that dimensional changes within the material obeyed ANSI/ADA standards (Nassar et al. 2017).

The limitation of this study was that the accuracy of the impression material was determined by studying the stability of the impression itself and that the setting expansion of the gypsum cast was not taken in account. However, the results confirmed the high precision of the experimental material, suggesting routine use of these materials for impression of fixed and removable partial dentures, complete dentures, precision attachments and implants. However, it is suggested to evaluate the effects of other disinfectant systems.

\section{Conclusions}

All three commercial and five experimental polyvinylsiloxane impressions exhibited linear expansion within the recommended range outlined by ANSI and ISO 4823; 2015 following 24hours of immersion, though extended immersion time amplified dimensional changes within all the groups. Therefore, following disinfection, the silicone impression materials must be poured within twenty-four hours.

\section{References}

Al Enazi TA and Naik AV. 2016. Disinfection of alginate and addition silicon rubber-based impression materials: Comparative analysis of $1 \%$ sodium hypochlorite and $2 \%$ glutaraldehyde. International journal of stomatology \& occlusion medicine 8(S1) 8:44-88.

Asopa SJ, Padiyar UN, Verma S, Suri P, Somayaji NS, and Radhakrishnan IC. 2020. Effect of heat sterilization and chemical method of sterilization on the polyvinyl siloxane impression material. A comparative study. Journal of family medicine and primary care 9:1348-1353. 10.4103/jfmpc.jfmpc_1122_19.

Association ANSiAD. 2007. ANNSI/ADA specification no. 19, dental elastomeric impression materials. Chicago: ADA. Journal of the American Dental Association 94:733-741.

Azevedo MJ, Correia I, Portela A, and Sampaio-Maia B. 2019. A simple and effective method for addition silicone impression disinfection. The journal of advanced prosthodontics 11:155-161. 10.4047/jap.2019.11.3.155 
216

217

218

219

220

221

222

223

224

225

226

227

228

229

230

231

232

233

234

235

236

237

238

239

240

241

242

243

244

245

246

247

248

249

250

251

252

253

254

255

256

257

258

Babiker GH, Nadia Khalifa B, Nasser M, and Alhajj B. 2018. Dimensional accuracy of alginate impressions using different methods of disinfection with varying concentrations. COMPENDIUM 39.

Carvalhal C, Mello J, Sobrinho LC, Correr AB, and Sinhoreti M. 2011. Dimensional change of elastomeric materials after immersion in disinfectant solutions for different times. J Contemp Dent Pract 12:252-258.

Correia-Sousa J, Tabaio AM, Silva A, Pereira T, Sampaio-Maia B, and Vasconcelos M. 2013. The effect of water and sodium hypochlorite disinfection on alginate impressions. Revista Portuguesa de Estomatologia, Medicina Dentária e Cirurgia Maxilofacial 54:8-12.

Din SU, Parker S, Braden M, and Patel M. 2018. The effects of cross-linking agent and surfactant on the tear strength of novel vinyl polysiloxane impression materials. Dental materials 34:e334-e343.

Guiraldo RD, Borsato TT, Berger SB, Lopes MB, Gonini-Jr A, and Sinhoreti MAC. 2012. Surface detail reproduction and dimensional accuracy of stone models: influence of disinfectant solutions and alginate impression materials. Brazilian dental journal 23:417-421.

ISO E. 4823: 2000-Dentistry: Elastomeric impression materials. European Committee for Standardization.

Iwasaki Y, Hiraguchi H, Iwasaki E, and Yoneyama T. 2016. Effects of immersion disinfection of agaralginate combined impressions on the surface properties of stone casts. Dental Materials Journal 35:45-50.

Khan MWU, Mushtaq MA. 2018. An overview of dental impression disinfection techniques-a literature review. JPDA 27:208.

Kumari N, and Nandeeshwar D. 2015. The dimensional accuracy of polyvinyl siloxane impression materials using two different impression techniques: An in vitro study. The Journal of the Indian Prosthodontic Society 15:211.

Lee DY, Oh YI, Chung KH, Kim KM, and Kim KN. 2004. Mechanism study on surface activation of surfactant-modified polyvinyl siloxane impression materials. Journal of applied polymer science 92:2395-2401.

Martin N, Martin M, and Jedynakiewicz N. 2007. The dimensional stability of dental impression materials following immersion in disinfecting solutions. Dental materials 23:760-768.

Nassar U, Flores-Mir C, Heo G, and Torrealba Y. 2017. The effect of prolonged storage and disinfection on the dimensional stability of 5 vinyl polyether silicone impression materials. The journal of advanced prosthodontics 9:182-187.

Samra RK, and Bhide SV. 2018. Comparative evaluation of dimensional stability of impression materials from developing countries and developed countries after disinfection with different immersion disinfectant systems and ultraviolet chamber. The Saudi dental journal 30:125-141.

Silva SMLMd, and Salvador MCG. 2004. Effect of the disinfection technique on the linear dimensional stability of dental impression materials. Journal of Applied Oral Science 12:244-249.

Thouati A, Deveaux E, Iost A, and Behin P. 1996. Dimensional stability of seven elastomeric impression materials immersed in disinfectants. The Journal of prosthetic dentistry 76:8-14.

Ud Din S, Hassan M, Khalid S, Zafar M, Ahmed B, and Patel M. 2018. Effect of surfactant's molecular weight on the wettability of vinyl polysiloxane impression materials after immersion disinfection. Materials Express 8:85-92. 10.1166/mex.2018.1413.

Peer) reviewing PDF | (2021:08:64727:2:1:NEW 7 Dec 2021) 
261

262 


\section{Table 1 (on next page)}

Composition of Experimental Polyvinyl siloxane impression materials 
Table 1:Composition of Experimental Polyvinyl siloxane impression materials ${ }^{2}$

\begin{tabular}{|c|c|c|c|c|c|}
\hline \multirow[b]{2}{*}{ Components } & \multicolumn{5}{|c|}{ Base Paste(Wt \%) } \\
\hline & Exp-A & Exp-B & Exp-C & Exp-D & $\begin{array}{l}\text { Exp- } \\
\text { E }\end{array}$ \\
\hline $\begin{array}{l}\text { Vinyl-terminated dimethylpolysiloxane } \\
\qquad(\mathrm{Mw} 62700)\end{array}$ & 39.90 & 39.90 & 37.95 & 37.46 & 36.98 \\
\hline Polymethylhydrosiloxane (Mw 2270) & 1.10 & 0.77 & 0.74 & 0.73 & 0.72 \\
\hline \multirow[t]{2}{*}{$\begin{array}{l}\text { Tetra-functional (dimethylsilyl) } \\
\text { orthosilicate (Mw 329) }\end{array}$} & - & 0.33 & 0.32 & 0.31 & 0.31 \\
\hline & \multicolumn{5}{|c|}{ Catalyst Paste(Wt \%) } \\
\hline Components & Exp-A & Exp-B & Exp-C & Exp-D & $\begin{array}{c}\text { Exp- } \\
\text { E }\end{array}$ \\
\hline $\begin{array}{l}\text { Vinyl-terminated dimethylpolysiloxane } \\
\qquad(\mathrm{Mw} 62700)\end{array}$ & 40.72 & 40.72 & 39.51 & 39.51 & 39.51 \\
\hline Platinum (0.05 M) & 0.06 & 0.06 & 1.27 & 1.27 & 1.27 \\
\hline Palladium $(<1 \mu \mathrm{m})$ & 0.23 & 0.23 & 0.22 & 0.22 & 0.22 \\
\hline Rhodasurf CET-2 & - & - & 2.00 & 2.50 & 3.00 \\
\hline
\end{tabular}




\section{Table 2 (on next page)}

Comparison of linear dimensional changes of impression materials stored in $1 \% \mathrm{NaOCl}$ disinfectant solution at 30 minutes 
1 Table 2: Comparison of linear dimensional changes of impression materials stored in $1 \% \mathrm{NaOCl}$

2 disinfectant solution at 30 minutes

\begin{tabular}{|c|c|c|c|c|c|}
\hline Materails & Mean & S.D & F (p-value) & Post -hoc Tukey test & p-value \\
\hline Aquasil & 0.058 & 0.029 & \multirow{8}{*}{$\begin{array}{l}3.657 \\
(0.005)\end{array}$} & Exp E vs Aquasil & 0.634 \\
\hline Extrude & 0.046 & 0.027 & & Exp E vs Extrude & 0.252 \\
\hline Elite & 0.021 & 0.028 & & Exp E vs Elite & 0.012 \\
\hline $\operatorname{Exp} A$ & 0.010 & 0.023 & & Exp E vs Exp A & 0.003 \\
\hline Exp B & 0.031 & 0.028 & & Exp E vs Exp B & 0.047 \\
\hline $\operatorname{Exp} C$ & 0.041 & 0.023 & & Exp E vs Exp C & 0.159 \\
\hline Exp D & 0.032 & 0.029 & & Exp E vs Exp D & 0.053 \\
\hline $\operatorname{Exp} E$ & 0.040 & 0.023 & & & \\
\hline
\end{tabular}

3 
Figure 1

Mean linear dimensional changes with Standard Deviation of commercilal and experimental PVS impression materials over the period of 24 hours following immersion in Distal water 


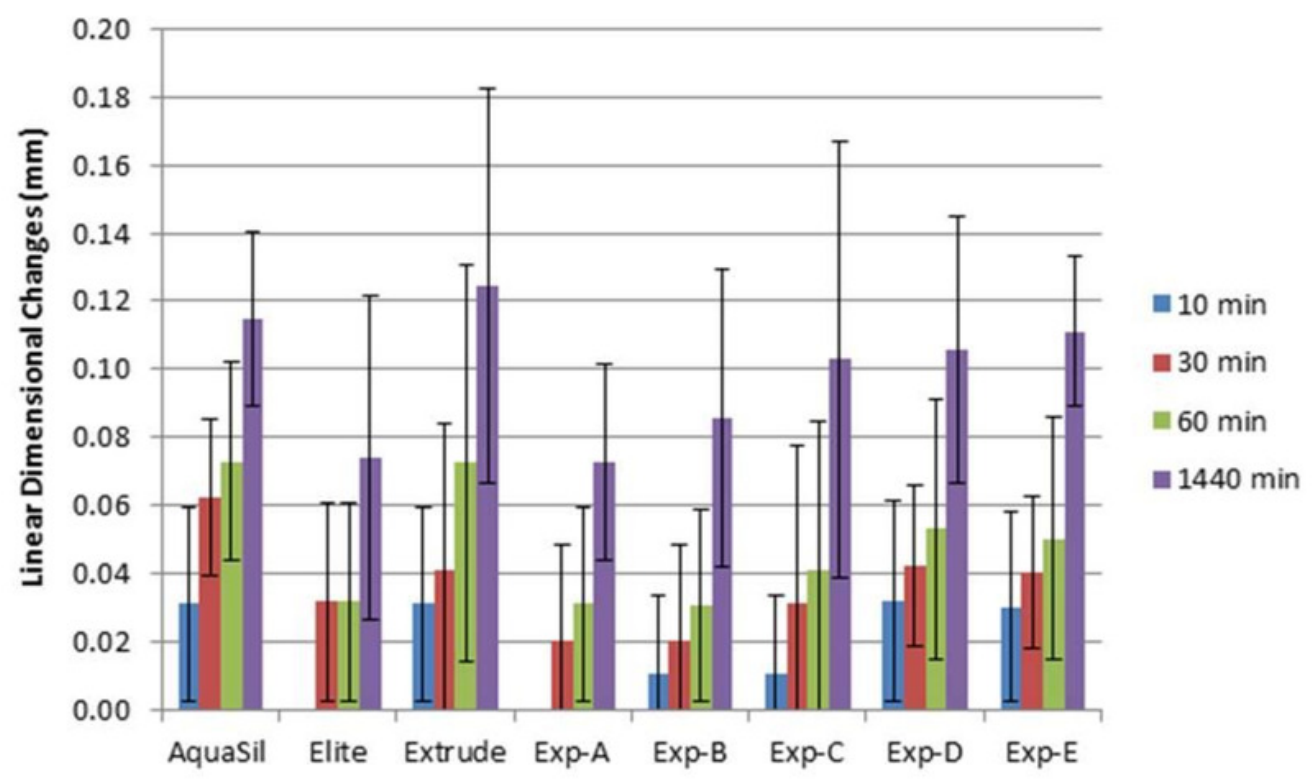


Figure 2

Mean linear dimensional changes with Standard Deviation of commercilal and experimental PVS impression materials over the period of 24 hours following immersion in $1 \% \mathrm{NaOCl}$ 


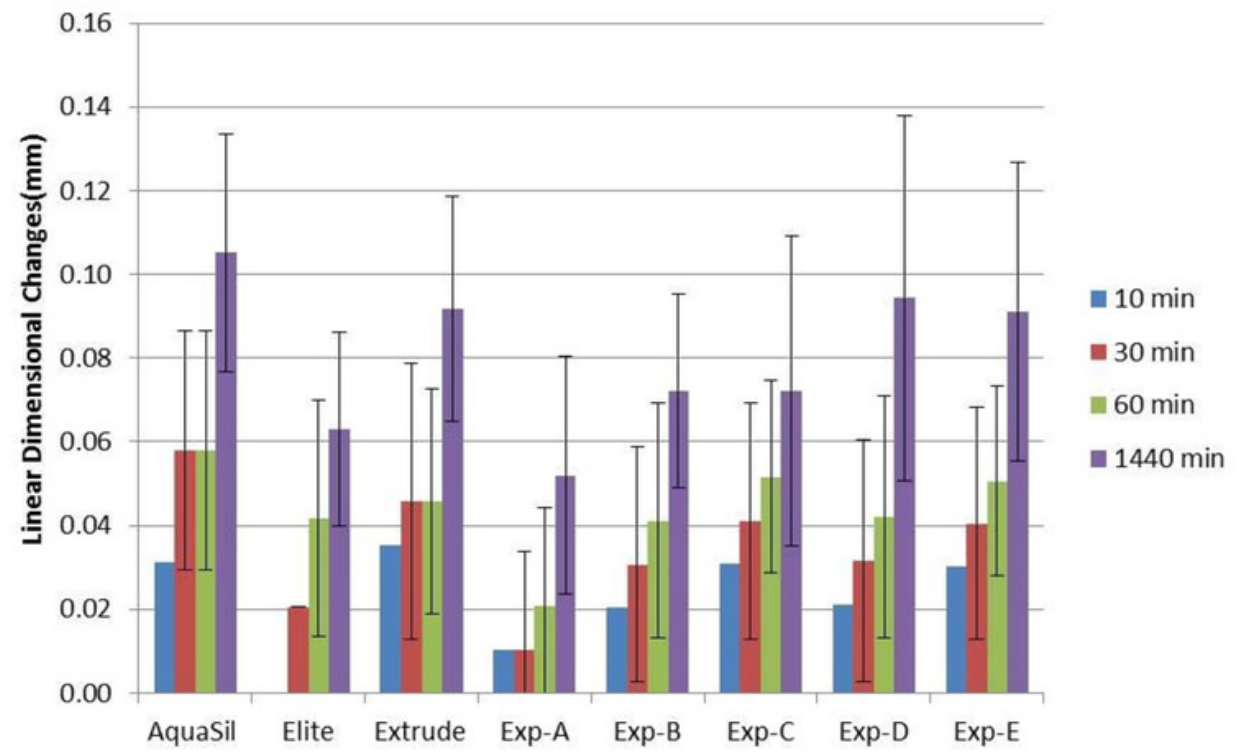

